\title{
OPTIMIZATION OF DYNAMIC AND MULTI-OBJECTIVE FLEXIBLE JOB-SHOP SCHEDULING BASED ON PARALLEL HYBRID ALGORITHM
}

\author{
Yang, X. P. * \& Gao, X. L.**\# \\ Nanchang Institute of Technology, Nanchang 330099, China \\ ${ }^{* *}$ College of Humanities, Shanghai University of Finance and Economics, Shanghai 200433, China \\ E-Mail: yxp1189@sina.com, gaoxiaoli007@163.com $\left({ }^{\#}\right.$ Corresponding author $)$
}

\begin{abstract}
This paper aims to develop a dynamic, real-time scheduling strategy under interference that can minimize the negative impact of interference on production scheduling without sacrificing the production efficiency. Taking the minimal cost and makespan as the objectives of the optimization function, the author put forward a parallel hybrid optimization algorithm for production rescheduling under interference, aiming to strike a balance between processing cost and scheduling disturbance. The benchmark test results show that the proposed algorithm achieved better accuracy than the NSGA-II and the AMOSA, and its accuracy has nothing to do with the distribution shape of the objective function or the continuity of the interference. In other words, the proposed algorithm enjoys strong computing stability. In the simulation tests, the proposed algorithm reached the global convergence state before reaching the maximum runtime, and consumed less time than the contrastive algorithms under the same problem scale. The research findings shed new light on the optimal scheduling of multi-objective FJSP under disturbance.

(Received, processed and accepted by the Chinese Representative Office.)
\end{abstract}

Key Words: Production Scheduling, Multi-Objective Scheduling, Parallel Hybrid Algorithm, Multiple Disturbances, Optimization, Simulation

\section{INTRODUCTION}

As an extension of the traditional job-shop scheduling problem, flexible job-shop scheduling problem (FJSP) assumes that one process can be implemented on multiple machines. Under this assumption, the path of each process needs to be selected from the available machines, which is close to the actual production environment. In the flexible job-shop, the production scheduling is usually optimized before the production and processing of a batch of jobs [1]. However, the actual production often suffers from various interferences, such as machine failure, delayed supply of raw materials, the insertion of new tasks, the cancelation of the original tasks and the change of processing priority [2-5]. Under these interferences, the original scheduling plan is no longer the optimal one, calling for a new plan that does not affect normal production [6, 7]. In other words, the job-shop production should be scheduled dynamically and in real time without affecting the multiple objectives of the original scheduling function (e.g. the minimal production cost, the shortest makespan, and the best production quality). Therefore, it is of great practical significance to develop a dynamic, realtime scheduling strategy under interference that can minimize the negative impact of interference on production scheduling without sacrificing the production efficiency $[8,9]$.

The existing studies on the FJSP mainly focus on the optimization of production scheduling under machine wear, makespan adjustment or lack of resources/funds, the production scheduling with the addition of new tasks, the cancellation of the original tasks and the change of processing priority [10], and the solving algorithms for production scheduling models under disturbance [11]. 
(1) Yang and Yang studied the impact of machine maintenance on production scheduling [12]. Rustogi and Strusevich explored the scheduling for minimal cost and minimal makespan under uncertain machines and personnel [13]. Li and Yuan investigated the optimization of production scheduling when it is allowed to reject the processing of jobs [14].

(2) The researchers qualitatively analysed the effects of interferences on production cost and production plan [15]. For instance, Liu et al. suppressed the impact of external disturbance on production scheduling through outsourcing [16]. Azizoglu and Alagöz remeasured the production cost with the total makespan and designed an optimized scheduling algorithm, aiming to disclose the influence of the addition of new tasks on the original scheduling plan [17]. Aktürk et al. probed deep into the adaptation of machine, resource and personnel to the optimized scheduling plan after the addition/cancellation of tasks [18].

(3) The solving algorithms mainly include precise optimization algorithms like dynamic programming, branch and bound method, etc. [19, 20], heuristic optimization algorithms like meta heuristic algorithm and heuristic hybrid rule algorithm [21, 22], as well as artificial intelligence algorithms like neural network algorithm, genetic algorithm and simulated annealing algorithms [23-25].

Despite certain research results, there are some defects in the existing studies on production scheduling under interference. For example, the makespan, a constant in current production scheduling models, actually varies with operation proficiency and machine wear [26]. There are few reports on the probability of machine failure or optimization of production scheduling under multiple interferences.

This paper mainly discusses the interference of the arrival of new orders to the original production scheduling in a FJSP. Taking the minimal cost and makespan as the objectives of the optimization function, the author put forward a parallel hybrid optimization algorithm for production rescheduling under interference, aiming to strike a balance between processing cost and scheduling disturbance. The research findings shed new light on the optimal scheduling of multi-objective FJSP under disturbance.

\section{MULTI-OBJECTIVE SCHEDULING ALGORITHM CONSIDERING MULTIPLE INTERFERENCES}

\subsection{Multi-objective optimization function}

The original objective function pursues the minimal cost. Let $n_{0}$ be the number of original jobs to be processed on a machine and $n_{N}$ be the number of new jobs to be added. Then, a total of $n_{0}+n_{N}$ jobs are to be processed on the machine. Let $p_{j}$ be the mean makespan of each job. Then, the actual makespan of each job under external interference can be expressed as:

$$
p_{j}^{A}=p_{j}\left(a+b t_{j}\right)
$$

where $a$ is a constant; $b$ is the processing deterioration rate; $t_{j}$ is the initial makespan.

Under the interference of the addition of a new job, the job is usually placed at the end of the processing sequence according to the traditional scheduling plans. This arrangement will push up the overall cost, due to the overlook of the processing priority and the optimal processing sequence. In this paper, the traditional arrangement is adjusted as follows. It is assumed that the minimal cost is the objective of the original scheduling plan:

$$
f_{1}=\sum C_{j}(\sigma)=\sum_{j=1}^{n_{O}+n_{N}}\left(p_{j}^{A}(\sigma)+t_{j}(\sigma)\right)
$$

Under system disturbance, the new processing cost $f_{2}$ can be expressed as: 


$$
f_{2}=\sum T_{j}(\sigma)=\sum_{j=1}^{n_{O}} \max \left\{C_{j}(\sigma)-\bar{C}_{j}, 0\right\}
$$

The above problem can be expressed below by the three-parameter method:

$$
1\left|p_{j}\left(a+b t_{j}\right)\right| \sum C_{j}(\sigma), \sum T_{j}(\sigma)=1\left|p_{j}\left(a+b t_{j}\right)\right| C_{\max }(\sigma)
$$

where $C_{\max }(\sigma)$ is the maximum makespan; $\sigma$ is the processing timetable; the value of $\sigma$ is independent of the processing sequence.

$$
\begin{gathered}
C_{\max }(\sigma)=t \prod_{j=1}^{n}\left(1+b p_{j}\right)+\frac{a}{b}\left(\prod_{j=1}^{n}\left(1+b p_{j}\right)-1\right) \\
\sum C_{j}(\sigma)=t \sum_{j=1}^{n} \prod_{i=1}^{n}\left(1+b p_{i}\right)+\sum_{j=1}^{n} \frac{a}{b}\left(\prod_{i=1}^{n}\left(1+b p_{i}\right)-1\right)
\end{gathered}
$$

\subsection{Design of parallel hybrid optimization algorithm}

In many studies, the non-dominated sorting genetic algorithm (NSGA-II) and archived multiobjective simulated annealing (AMOSA) algorithm are adopted to solve the production scheduling problem under interference. The NSGA-II enjoys fast convergence and high diversity, but may converge prematurely when dealing with complex problems. By contrast, the AMOSA can effectively avoid the local optimum trap during the search process, and make the result as close as possible to the effective front of the optimal solution.

In light of the above, this paper optimizes the NSGA-II with the AMOSA, and proposes an innovative parallel hybrid optimization algorithm. The calculation flow of the proposed algorithm is illustrated in Fig. 1. The specific steps of the algorithm are as follows.

(1) Parameters were initialized and the initial population $P_{N}$ was generated. The initial parameters include population size pop, archive size, number of climbs, etc.

The coding method of the proposed algorithm can be explained as:

$$
\begin{aligned}
& x_{i k}=\left\{x_{i k}^{1}, x_{i k}^{2}, \ldots, x_{i k}^{n}, x_{i k}^{n+1}, x_{i k}^{n+2}, \ldots, x_{i k}^{2 n}\right\} \\
& i=1,2, \ldots \text { pop } ; k=1,2, \ldots, \text { iterations }
\end{aligned}
$$

where $x_{i k}$ is individual $i$ in the population of the $k^{\text {th }}$ generation. Note that $x_{i k}{ }^{1} \sim x_{i k}{ }^{n}$ belong to the product sequencing part $(\pi)$, while $x_{i k}^{n+1} \sim x_{i k}^{2 n}$ belong to the compressed vector part $(y)$. The solution efficiency of the $\pi$ part was improved by permutation encoding, and the genetic code was translated into the processing order during the fitness calculation; the $y$ part was transformed according to the following formula:

$$
y_{i}=\left\{\begin{array}{l}
0, \quad 0 \leq x^{j} \leq 0.5 \\
u^{\pi[j-n]}, \quad 0.5<x^{j} \leq 1
\end{array} \quad j=n+1, n+2, \ldots, 2 n\right.
$$

(2) The main parameters (e.g. initial temperature) were determined. The calculated values of the optimal total makespan and the optimal total cost were regarded as the ideal points, while those of the actual total makespan and the actual total cost were considered as the actual target points. Then, the distance between each pair of ideal point and actual point was determined, and the maximum distance was denoted as $\Delta \max$. Then, the initial temperature function $T_{0}$ can be expressed as:

$$
T_{0}=-|\Delta \max | / \ln p r
$$

where $p r=0.1$ is the initial acceptance probability. 


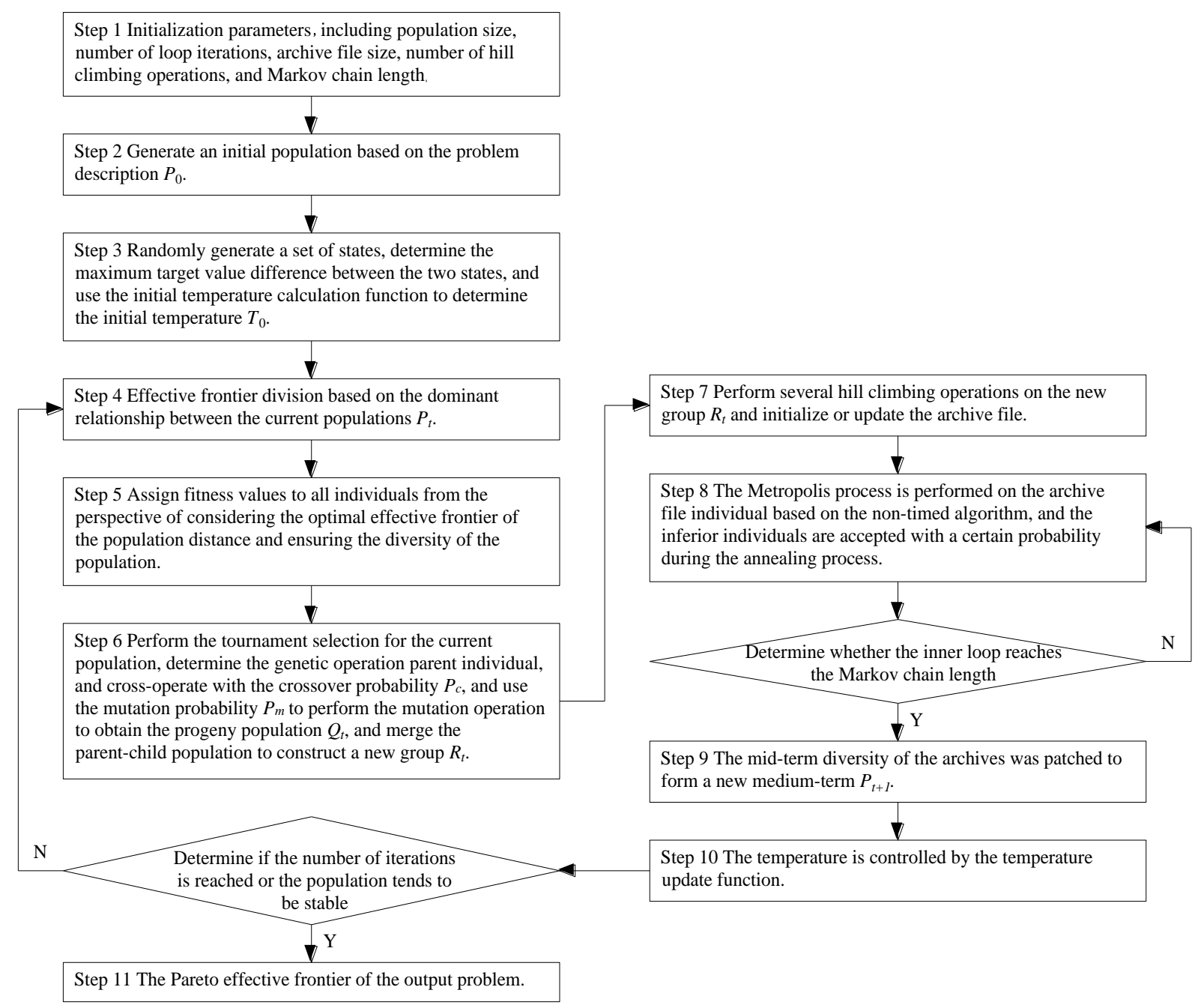

Figure 1: Calculation flow of the proposed algorithm.

(3) The initial population was divided by fitness and effective front. The individuals in the population were mapped to the non-dominated fronts according to their dominance relationship. For fronts $F_{i}$ and $F_{j}$, the individuals in $F_{i}$ dominate over those in $F_{j}$. The inverse is also true.

(4) The individuals in the population were assigned with fitness and sorted out, and the parent population $P_{p}$ was determined.

(5) The parent population was subjected to crossover and mutation, forming the child population $p_{0}$.

During the crossover, the unrepeated genes in the $\pi$ part in Eq. (7) were subjected to single-point crossover, while the repeated ones were not subjected to crossover; all the genes in the y part were subjected to crossover.

During the mutation, the $x_{i k}{ }^{n}$ in the $\pi$ part were mutated into other genes, forming the candidate solutions, while the $y$ part was subjected to traditional mutation of $0-1$ conversion.

(6) The original and the child populations were combined, and the excellent individuals were selected from the merged population to form a new population $R_{N}$.

(7) The original population was subjected to mountain climbing operation and went through the Metropolis process. The archive file Arch was updated after each iteration.

(8) The new population in step (6) and Arch were combined into the next generation population $P_{N+1}$ and the effective Pareto solution of the algorithm was finally outputted. 


\section{BENCHMARK TEST RESULTS}

The proposed algorithm was verified by the standard test functions ZDT 1 5:

$$
\begin{aligned}
& \min \left(f_{1}\left(x_{1}\right), f_{2}(x)\right) \\
& \text { s.t. } f_{2}(x)=g\left(x_{2}, \ldots, x_{m}\right) h\left(f_{1}\left(x_{1}\right), g\left(x_{2}, \ldots, x_{m}\right)\right)
\end{aligned}
$$

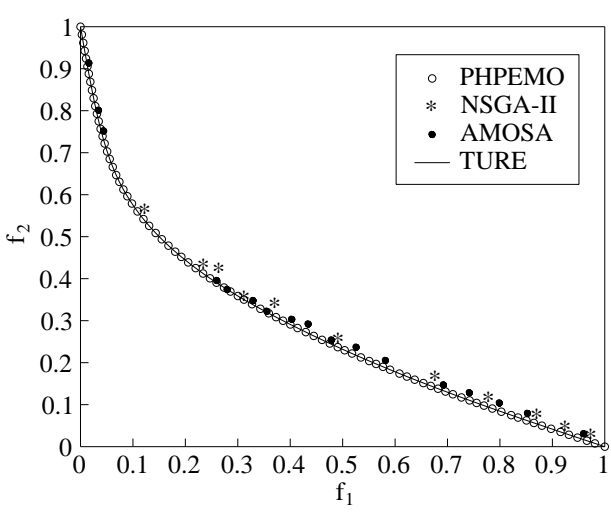

a) ZDT-1

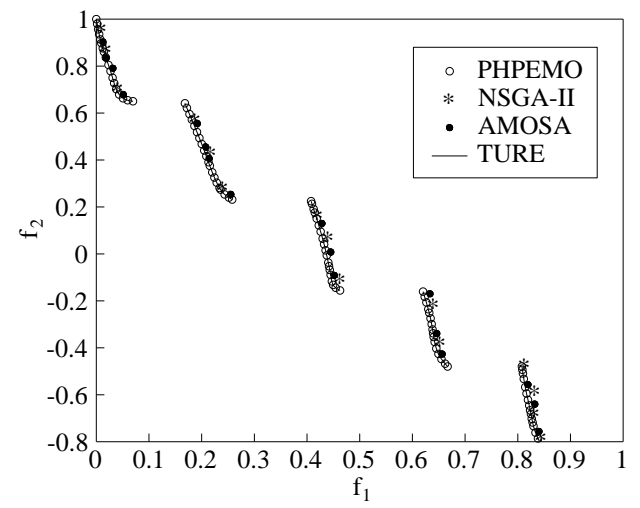

c) ZDT-3

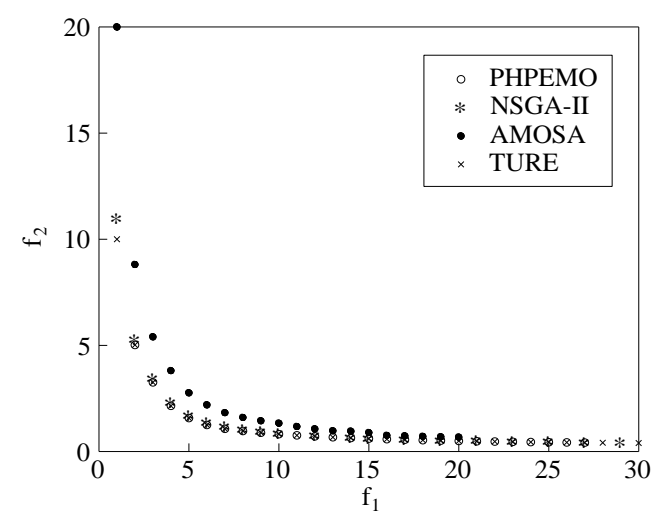

e) ZDT-5

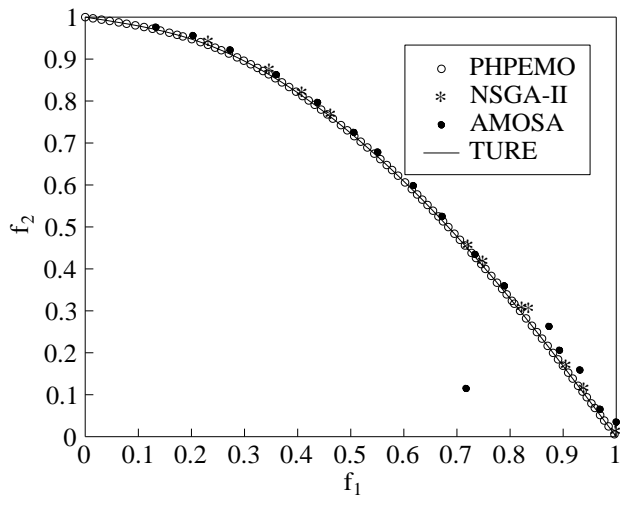

b) ZDT-2

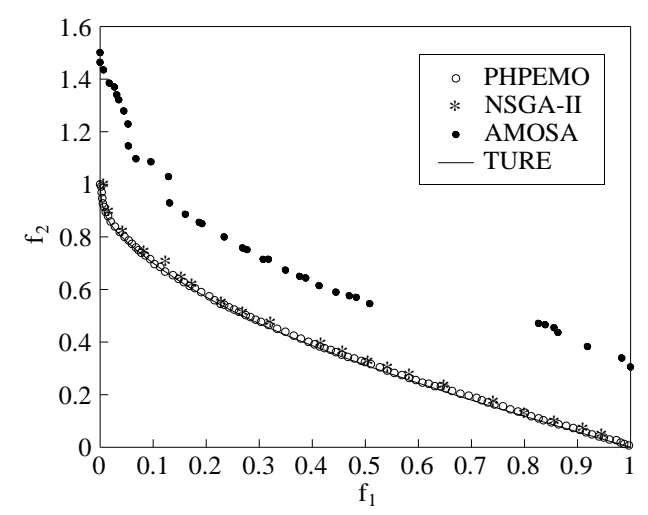

d) ZDT-4

Figure 2: Results of benchmark test.

ZDT 1 4 are continuous variable functions while ZDT-5 is discrete variable function. ZDT 1 3 all have 30 dimensions, with ZDT-1 being convex, ZDT-2 being concave and ZDT-3 being sectional-continuous; ZDT-4 has 10 dimensions and does well in the testing of the efficiency of multimodality processing; ZDT-5 has 80 dimensions and a spoofed Pareto front.

According to the nature of the test functions ZDT 1 5, the distance between a location and the effective front in the search space is negatively correlated with the number of 
individuals at the location. Thus, these functions can be applied to check if the proposed algorithm target dependence.

Here, the standard test functions ZDT 1 5 are adopted to verify the proposed algorithm, the NSGA-II and the AMOSA. The results are shown in Fig. 2 blow. It can be seen that the result of each algorithm was independent of the structure and shape of the objective function, but the three algorithms differed greatly in the calculated results.

Below is a comparison between the NSGA-II and the AMOSA. For ZDT-2, the NSGA-II achieved a poorer accuracy than the AMOSA. With the growing difficulty of the test functions, the accuracy of the AMOSA started to decline. For ZDT-3 and ZDT-4, the AMOSA found the solutions on the optimal effective front in the search space. By contrast, the NSGA-II results were all close to the optimal effective front in ZDT 3 5. Overall, the NSGA-II outperformed the AMOSA.

For ZDT 1 5, the proposed algorithm always outshined the NSGA-II and the AMOSA in terms of accuracy. Besides, the accuracy of the proposed algorithm has nothing to do with the disturbances of the objective function (distribution shape and continuity), evidence to a high computing stability. In addition, the proposed algorithm found the optimal Pareto front for all test functions other than ZDT-4. Thus, it is proved that the proposed algorithm has better accuracy and stability than the two contrastive algorithms.

\section{SIMULATION TESTS AND RESULTS ANALYSIS}

Several simulation tests were carried out to verify the effectiveness and superiority of the proposed algorithm for the production scheduling under disturbance. First, the proposed algorithm was contrasted with the NSGA-II in the population evolution at different number of iterations. The calculated results are shown in Fig. 3 below.

As shown in Fig. 3 a, the two algorithms had the same initial populations at the beginning. As shown in Fig. $3 \mathrm{~b}$, the proposed algorithm gradually exceeded the NSGA-II in the optimization performance, as some of its individuals were closer to the ideal points. Starting from the $45^{\text {th }}$ iteration to the $200^{\text {th }}$ iteration, the proposed algorithm enjoyed a huge edge over the NSGA-II in superiority. Fig. 3 clearly demonstrates the fast convergence and high search accuracy of the proposed algorithm.

Fig. 4 compares the computing time of the proposed algorithm, the NSGA-II and the AMOSA under different problem scales. It can be seen that the proposed algorithm reached the global convergence state before reaching the maximum runtime, and consumed the least time under the same problem scale. The results in this figure manifest the superiority of the proposed algorithm in the fast search for the optimal Pareto front.

Fig. 5 presents the convergence curves of the three algorithms at different number of iterations. It is clear that the NSGA-II tended to be stable at the $38^{\text {th }}$ iteration, with a large distance to the ideal point. This means the NSGA-II exhibited an obvious premature phenomenon. By contrast, the proposed algorithm reached the equilibrium state as late as the $100^{\text {th }}$ iteration, with a small distance to the ideal distance, indicating that the algorithm overcomes the premature convergence to local optimal solutions of the traditional algorithms.

This is attributable to the improvement of the NSGA-II with the AMOSA.

Fig. 6 shows the Pareto effective fronts calculated by the three algorithms when the jobshop processes 20, 100 and 200 jobs. It can be seen that the calculated results of the proposed algorithm were closer to the ideal point than those of the contrastive algorithms, and the Pareto effective front of the proposed algorithm was closer to the lower left than that of the contrastive algorithms. With the increase in the number of jobs, the quality of the Pareto effective front outputted by the proposed algorithm was on the rise. 


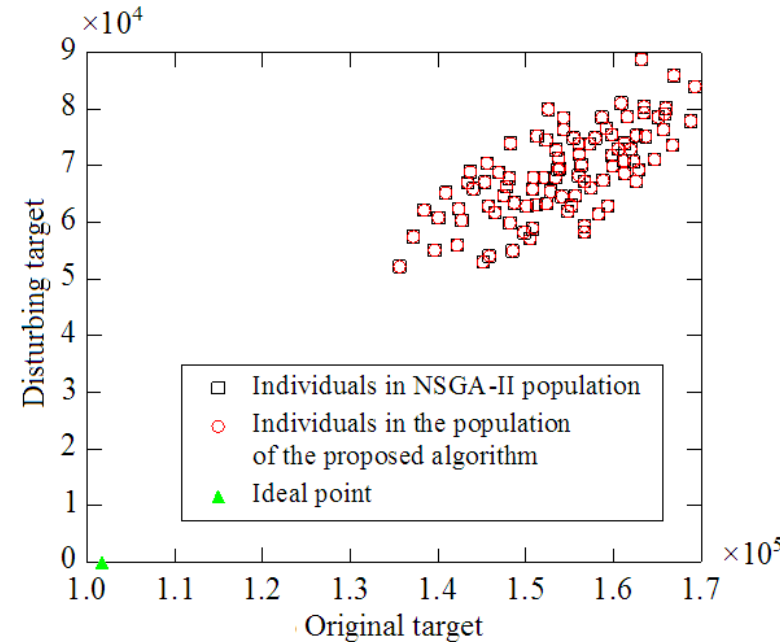

a) initial population

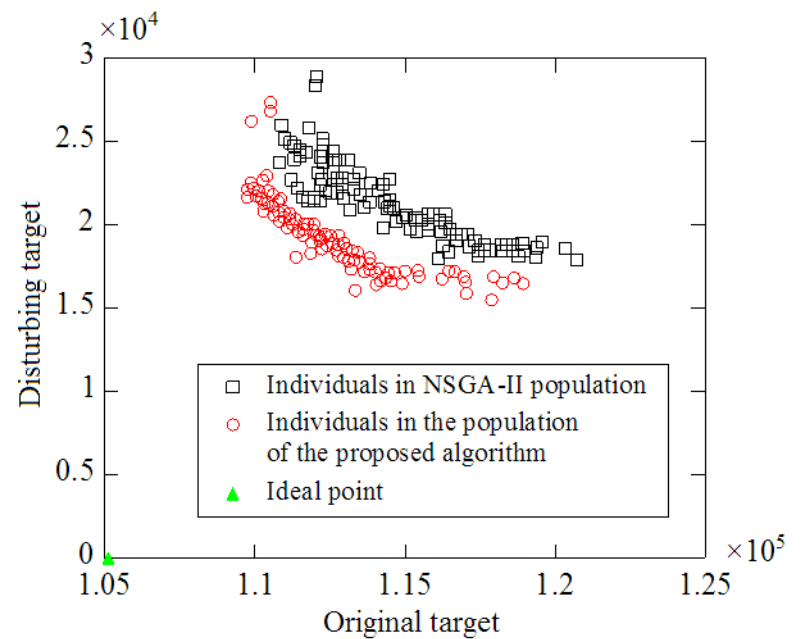

c) the $45^{\text {th }}$ generation population

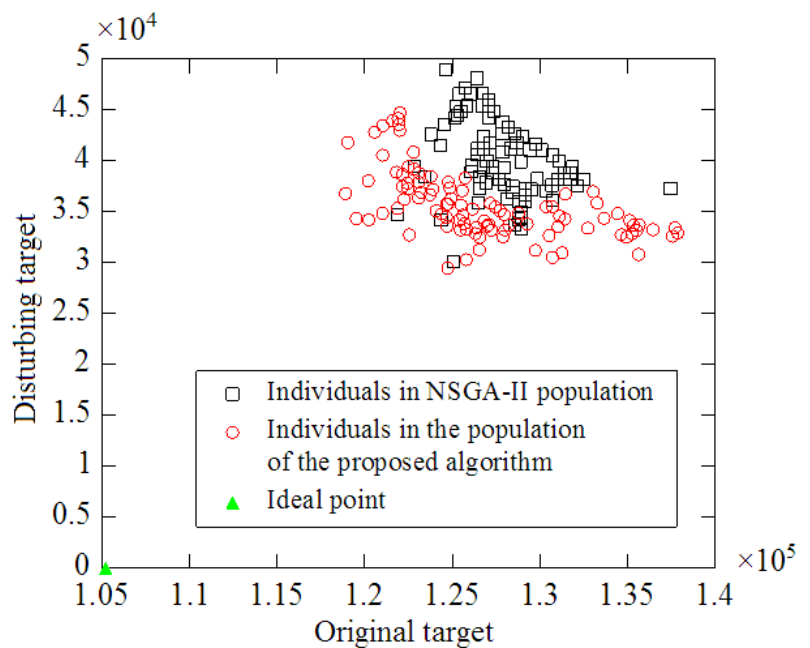

b) the $15^{\text {th }}$ generation population

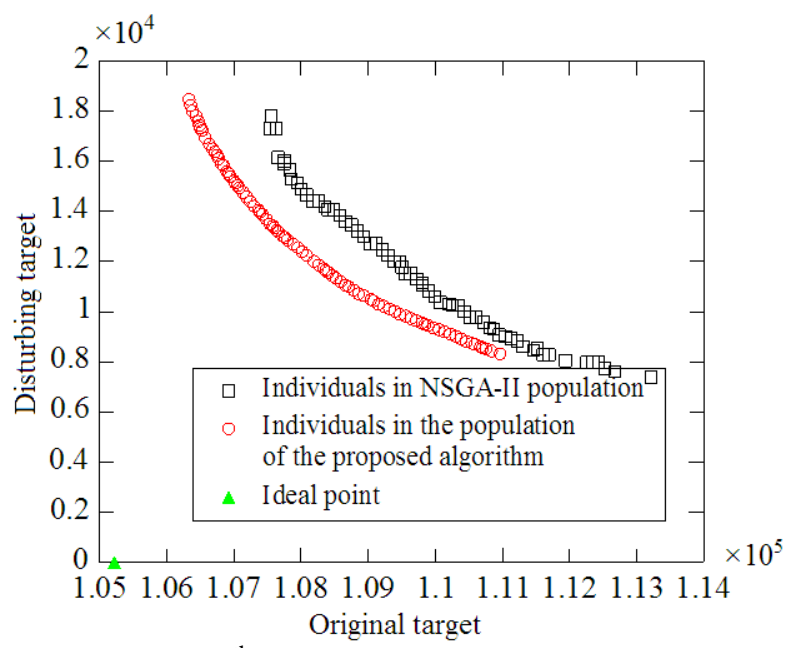

d) the $200^{\text {th }}$ generation population

Figure 3: Population evolution of the proposed algorithm and the NSGA-II at different number of iterations.

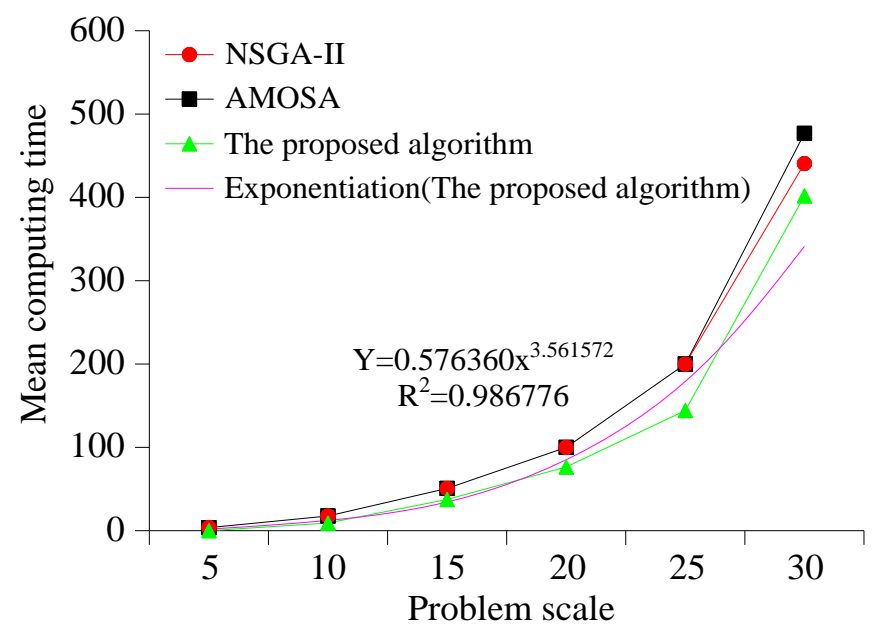

Figure 4: Computing time of the three algorithms under different problem scales. 


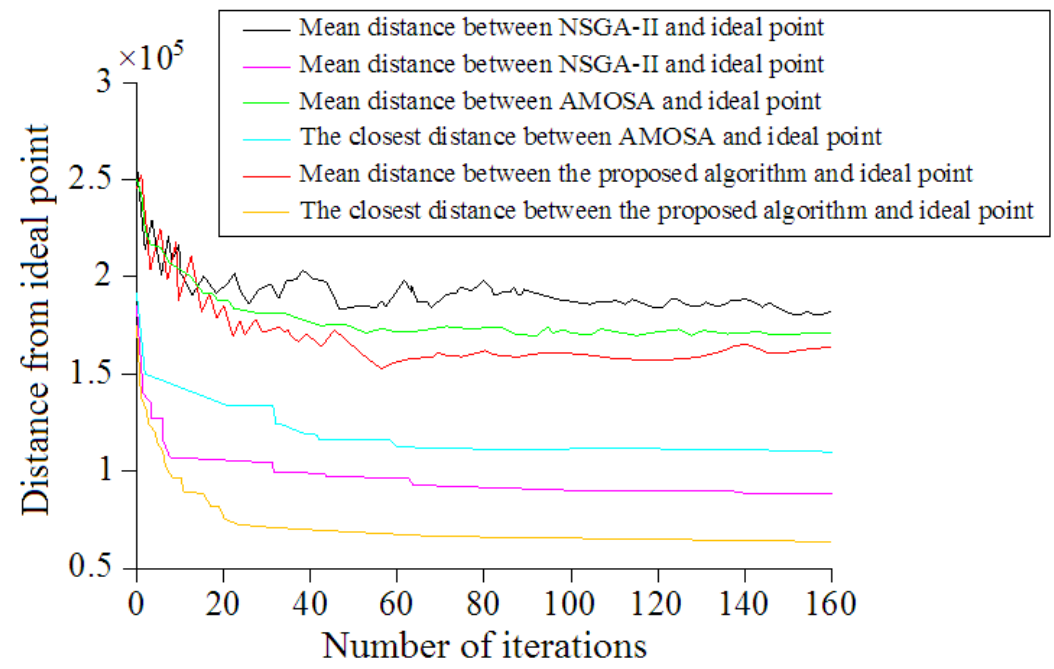

Figure 5: Convergence curves of the three algorithms at different number of iterations.

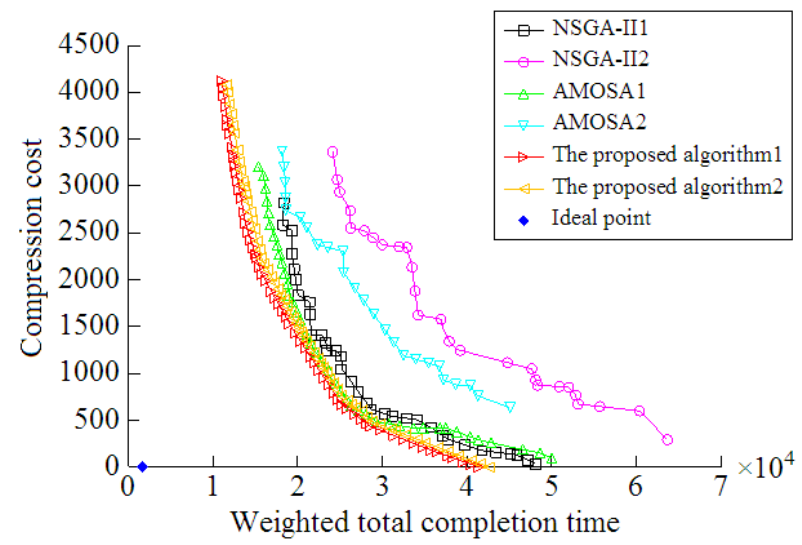

a) 20 jobs

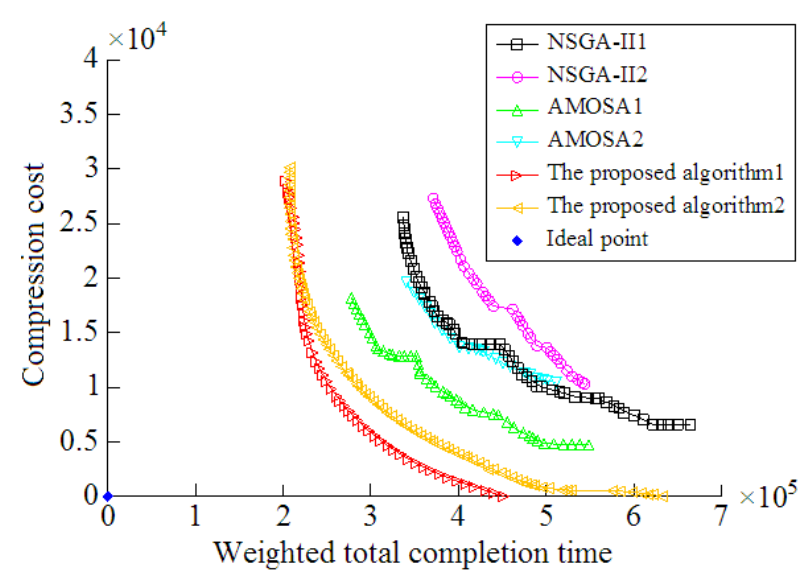

c) 200 jobs

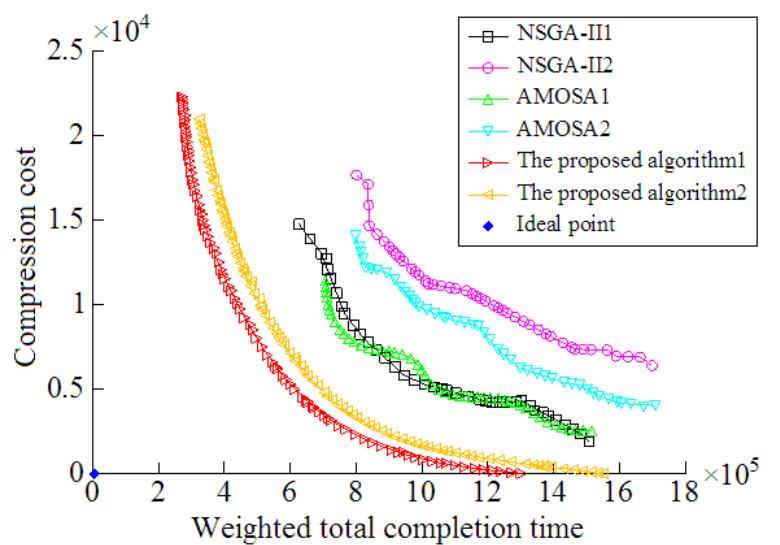

b) 100 jobs

Figure 6: Pareto effective fronts of the three algorithms at different number of jobs.

\section{CONCLUSIONS}

Taking the minimal cost and makespan as the objectives, this paper establishes a multiobjective optimization scheduling model for the FJSP, and puts forward a parallel hybrid optimization algorithm for production rescheduling under interference, aiming to strike a balance between processing cost and scheduling disturbance. The research findings are as follows: 
(1) The proposed algorithm optimizes the premature convergence of the NSGA-II by the AMOSA, and relies on external archiving to increase the diversity of the initial population and excellent child populations. The benchmark test results show that the proposed algorithm achieved better accuracy than the NSGA-II and the AMOSA, and its accuracy has nothing to do with the distribution shape of the objective function or the continuity of the interference. In other words, the proposed algorithm enjoys strong computing stability.

(2) In the simulation tests, the proposed algorithm reached the global convergence state before reaching the maximum runtime, and consumed less time than the contrastive algorithms under the same problem scale. In addition, the proposed algorithm could overcome the premature convergence to local optimal solutions of the traditional algorithms.

\section{ACKNOWLEDGEMENTS}

This paper is supported by National Natural Science Foundation of China (51567018), Science and Technology Project Founded by the Education Department of Jiangxi Province (GJJ151131), Nature Science Foundation of Jiangxi Province, China (20171BAB216036) and National Natural Science Foundation of China (21603093, 51765044)

\section{REFERENCES}

[1] Cowling, P.; Johansson, M. (2002). Using real time information for effective dynamic scheduling, European Journal of Operational Research, Vol. 139, No. 2, 230-244, doi:10.1016/s0377-2217(01)00355-1

[2] Aytug, H.; Lawley, M. A.; McKay, K.; Mohan, S.; Uzsoy, R. (2005). Executing production schedules in the face of uncertainties: a review and some future directions, European Journal of Operational Research, Vol. 161, No. 1, 86-110, doi:10.1016/j.ejor.2003.08.027

[3] Burdett, R. L.; Kozan, E. (2015). Techniques to effectively buffer schedules in the face of uncertainties, Computers \& Industrial Engineering, Vol. 87, 16-29, doi:10.1016/ j.cie.2015.04.024

[4] Glazebrook, K. D. (1984). Scheduling stochastic jobs on a single machine subject to breakdowns, Naval Research Logistics Quarterly, Vol. 31, No. 2, 251-264, doi:10.1002/nav.3800310207

[5] Wang, D.-J.; Wang, J.-J.; Liu, C.-L.; Wang, Y.-Z. (2015). Disruption management for multiple new orders in production scheduling with deteriorating processing time, Systems Engineering Theory \& Practice, Vol. 35, No. 2, 368-380

[6] Cai, X.; Sun, X.; Zhou, X. (2003). Stochastic scheduling with preemptive-repeat machine breakdowns to minimize the expected weighted flow time, Probability in the Engineering and Informational Sciences, Vol. 17, No. 4, 467-485, doi:10.1017/s0269964803174037

[7] Kellerer, H.; Rustogi, K.; Strusevich, V. A. (2013). Approximation schemes for scheduling on a single machine subject to cumulative deterioration and maintenance, Journal of Scheduling, Vol. 16, No. 6, 675-683, doi:10.1007/s10951-012-0287-8

[8] He, W.; Sun, D.-H. (2013). Scheduling flexible job shop problem subject to machine breakdown with route changing and right-shift strategies, International Journal of Advanced Manufacturing Technology, Vol. 66, No. 1-4, 501-514, doi:10.1007/s00170-012-4344-4

[9] Tang, H. Y.; Tang, C. H.; Zhao, C. L. (2010). A preemptive-resume stochastic scheduling model with disruption, Systems Engineering - Theory \& Practice, Vol. 30, No. 4, 751-757

[10] Gordon, V. S.; Potts, C. N.; Strusevich, V. A.; Whitehead, J. D. (2008). Single machine scheduling models with deterioration and learning: handling precedence constraints via priority generation, Journal of Scheduling, Vol. 11, No. 5, Paper 357, doi:10.1007/s10951-008-0064-x

[11] Hu, X.-P.; Zhang, Y.; Ding, Q.-L.; Wang, X.-P. (2008). Review on disruption management model and its algorithm, Systems Engineering - Theory \& Practice, Vol. 28, No. 10, 40-46

[12] Yang, S.-J.; Yang, D.-L. (2010). Minimizing the total completion time in single-machine scheduling with aging/deteriorating effects and deteriorating maintenance activities, Computers \& Mathematics with Applications, Vol. 60, No. 7, 2161-2169, doi:10.1016/j.camwa.2010.08.003 
[13] Rustogi, K.; Strusevich, V. A. (2012). Single machine scheduling with general positional deterioration and rate-modifying maintenance, Omega, Vol. 40, No. 6, 791-804, doi:10.1016/ j.omega.2011.12.007

[14] Li, S.; Yuan, J. (2010). Parallel-machine scheduling with deteriorating jobs and rejection, Theoretical Computer Science, Vol. 411, No. 40-42, 3642-3650, doi:10.1016/j.tcs.2010.06.008

[15] Gürel, S.; Cincioğlu, D. (2015). Rescheduling with controllable processing times for number of disrupted jobs and manufacturing cost objectives, International Journal of Production Research, Vol. 53, No. 9, 2751-2770, doi:10.1080/00207543.2014.980452

[16] Liu, F.; Wang, J.-J.; Chen, H.; Yang, D.-L. (2014). Machine scheduling with outsourcing: coping with supply chain uncertainty with a second supplying source, The International Journal of Logistics Management, Vol. 25, No. 1, 133-159, doi:10.1108/ijlm-12-2012-0142

[17] Azizoglu, M.; Alagöz, O. (2005). Parallel-machine rescheduling with machine disruptions, IIE Transactions, Vol. 37, No. 12, 1113-1118, doi:10.1080/07408170500288133

[18] Aktürk, M. S.; Atamtürk, A.; Gürel, S. (2010). Parallel machine match-up scheduling with manufacturing cost considerations, Journal of Scheduling, Vol. 13, No. 1, 95-110, doi:10.1007/s10951-009-0111-2

[19] Buyurgan, N.; Mendoza, A. (2006). Performance-based dynamic scheduling model for flexible manufacturing systems, International Journal of Production Research, Vol. 44, No. 7, 1273 1295, doi: $10.1080 / 00207540500412016$

[20] Li, Q.-Y.; Wang, B.; Wang, X.-M. (2011). Predictable scheduling approach for single machine subject to random machine breakdown, Systems Engineering - Theory \& Practice, Vol. 31, No. $12,2387-2393$

[21] Li, Z. P.; Zhang, J. L.; Zhang, H. J.; Hua, G. W. (2017). Optimal selection of movable shelves under cargo-to-person picking mode, International Journal of Simulation Modelling, Vol. 16, No. 1, 145-156, doi:10.2507/IJSIMM16(1)CO2

[22] Nearchou, A. C. (2010). Scheduling with controllable processing times and compression costs using population-based heuristics, International Journal of Production Research, Vol. 48, No. 23, 7043-7062, doi:10.1080/002075409034333874

[23] Yu, H. (2018). Numerical simulation of European option payoff based on stochastic differential delay equations, Mathematical Modelling of Engineering Problems, Vol. 5, No. 2, 102-107, doi: $10.18280 / \mathrm{mmep} .050207$

[24] Sun, Y.; Chen, T. G. (2012). Research on disruption management for multi-objective problem and its solution by genetic algorithm, Advanced Materials Research, Vol. 433-440, 5040-5044, doi:10.4028/www.scientific.net/amr.433-440.5040

[25] Bandyopadhyay, S.; Saha, S.; Maulik, U.; Deb, K. (2008). A simulated annealing-based multiobjective optimization algorithm: AMOSA, IEEE Transactions on Evolutionary Computation, Vol. 12, No. 3, 269-283, doi:10.1109/tevc.2007.900837

[26] Shabtay, D.; Steiner, G. (2007). A survey of scheduling with controllable processing times, Discrete Applied Mathematics, Vol. 155, No. 13, 1643-1666, doi:10.1016/j.dam.2007.02.003 